\title{
Effects of Pathological Flow on Pulmonary Artery Endothelial Production of Vasoactive Mediators and Growth Factors
}

\author{
Min Li ${ }^{a}$, $\quad$ Kurt R. Stenmark ${ }^{a}$ Robin Shandas ${ }^{a, b}$ Wei Tan ${ }^{a, b}$ \\ ${ }^{a}$ Department of Pediatrics and CVP Research, University of Colorado at Denver and Health Sciences Center, \\ Denver, Colo., and ${ }^{b}$ Department of Mechanical Engineering, University of Colorado at Boulder, Boulder, Colo., USA
}

\section{Key Words}

Pulmonary hypertension $\cdot$ Shear stress $\cdot$ Nitric oxide $\cdot$

Endothelin $\cdot$ Vascular tone $\cdot$ Vascular remodeling

\begin{abstract}
Background: Alterations in pulmonary blood flow are often associated with the initiation and progression of pulmonary vascular disease. However, the cellular mechanisms involved in mediating flow effects in the pulmonary circulation remain unclear. Depending on the disease condition, flow may be extremely low or high. We therefore examined effects of pathologically low and high flow on endothelial production of factors capable of affecting pulmonary vascular tone and structure as well as on potential underlying mechanisms. Methods: Flow effects on pulmonary endothelial release of NO, PGF 1 a, ET-1 and $\mathrm{TxB}_{2}$, on expression of total and phosphorylated eNOS as well as Akt, and on VEGF were examined. Additionally, in a coculture system, effects of flow-exposed endothelial cells on smooth muscle (SM) proliferation and contractile protein were studied. Results: Compared to physiological flow, pathologically high and low flow attenuated endothelial release of $\mathrm{NO}$ and $\mathrm{PGF}_{1 \mathrm{a}}$, and enhanced release of ET-1. Physiological flow activated the Akt/eNOS pathway, while pathological flow depressed it. Pathologically high flow altered VE-cadherin expression. Pathologically high flow on the endothelium upregulated $\alpha$-SM-actin and SMMHC without affecting SM proliferation. Conclusion: Physi-
\end{abstract}

ological flow leads to production of mediators which favor vasodilation. Pathological flow alters the balance of mediator production which favors vasoconstriction.

Copyright $\odot 2009$ S. Karger AG, Basel

\section{Introduction}

Alterations in pulmonary blood flow are thought to be a critical factor in the initiation and exacerbation of pulmonary vascular diseases [1-4]. Changes in the flow magnitude are observed in both acute and chronic forms of pulmonary hypertension [3]. Studies in animals and humans have shown that high blood flow can lead to the development of pulmonary hypertension [5-7]. Increases in blood flow through the creation of systemic to pulmonary shunts are often used to induce pulmonary hypertension in animals [8]. Significantly increased blood flow and flow shear stress is also found in patients with congenital heart diseases having a systemic to pulmonary communication such as patent ductus arteriosus or atrial and ventricular septal defects, conditions which often lead to pulmonary hypertension. Peak flow shear stresses in these conditions may reach levels far above physiological shear stress $\left(>80 \mathrm{dyn} / \mathrm{cm}^{2}\right)$ [9]. Additionally, reports have shown that patients with severe pulmonary hypertension are characterized by reduced blood flow and low shear stress (5-8 dyn $/ \mathrm{cm}^{2}$ ) compared to normal flow and

\section{KARGER}

(C) 2009 S. Karger AG, Basel

Fax +4161306 1234 E-Mail karger@karger.ch www.karger.com www.karger.com/jvr
Dr. Wei Tan

Department of Pediatrics, University of Colorado Health Science Center 4200 E Ninth Ave, Room 3408

Denver, CO 80262 (USA)

Tel. +1 303315 0693, Fax +1 303492 3498, E-Mail wei.tan-1@colorado.edu 
physiological shear stress $\left(15-20 \mathrm{dyn} / \mathrm{cm}^{2}\right)$ in normotensive patients [4]. Thus, both increased and decreased flow may induce adverse responses in the pulmonary vasculature, as demonstrated in the banded left pulmonary artery model [10]. However, despite these observations, few studies have attempted to define the mechanisms underlying how the magnitude of flow regulates endothelial function in the pulmonary circulation.

Previous studies have established that shear stress is an important mechanical factor that regulates endothelial structure, function and growth. Numerous investigations, primarily in the systemic circulation, have focused on physiological shear stress $\left(10-25 \mathrm{dyn} / \mathrm{cm}^{2}\right)$ and relatively low shear stress $\left(<5 \mathrm{dyn} / \mathrm{cm}^{2}\right)$ as conditions relevant to development of atherosclerosis. Generally, it is believed that smooth, laminar physiological shear stress provides atheroprotective effects, while turbulent, oscillatory or low flow shear stress $\left(-4\right.$ to $\left.5 \mathrm{dyn} / \mathrm{cm}^{2}\right)$ stimulates atherosclerosis formation [11]. Physiological flow shear stress $\left(10-25 \mathrm{dyn} / \mathrm{cm}^{2}\right)$ serves as an important regulator of pathophysiologically relevant gene expression in endothelial cells [12-15]. Interestingly, despite the vast number of studies in endothelial mechanobiology, surprisingly little is known about the effect of pathologically high flow shear stress on the structure and function of the pulmonary vasculature despite the fact that shear stresses often above $70 \mathrm{dyn} / \mathrm{cm}^{2}$ levels have been observed in hypertension conditions [11].

Given that the flow magnitude plays a role in the pathogenesis and progression of pulmonary diseases, we sought to investigate the effects of both pathologically high and low shear, compared to physiological shear, on pulmonary endothelial production of vasodilating and vasoconstricting factors. We also wanted to examine the effects of said changes on smooth muscle cell (SMC) phenotype by examining contractile protein and proliferation changes in SMCs cocultured with endothelial cells exposed to varying shear conditions. To test this, we have designed hemodynamic flow conditions to encompass both low and high end of pathological flow shear stresses ranging from 5 to $120 \mathrm{dyn} / \mathrm{cm}^{2}$ and studied their influences on pulmonary arterial endothelial cells (PAECs) and pulmonary arterial SMCs (PASMCs).

\section{Materials and Methods}

\section{Cell Culture}

Bovine PAECs and bovine PASMCs were isolated from neonatal calves as previously described $[16,17]$. PAECs were maintained in a growth medium (D-Valine MEM medium; Mediatech Inc.,
Herndon, Va., USA) containing 20\% fetal bovine serum (FBS; Gemini Bio-products, West Sacramento, Calif., USA), 2\% L-glutamine (Invitrogen, Carlsbad, Calif., USA) and 5\% gentamicin (Invitrogen/Gibco; Carlsbad, Calif., USA). PASMCs were maintained in Dulbecco's modification of eagle's medium (DMEM; Cellgro, Herndon, Va., USA) containing 10\% FBS and 5\% gentamicin (MP Biomedicals, LLC, Solon, Ohio, USA). Cells at passages $4-8$ were used for all experiments.

Experimental Protocols for Flow Shear Stress Studies

To examine the response of PAEC monolayer to flow conditions, plain microscope slides were coated with $25 \mu \mathrm{g} / \mathrm{ml}$ fibronectin. Then, PAECs at a concentration of $4.0 \times 10^{5} / \mathrm{ml}$ were seeded on the fibronectin-coated slides and grown to confluence. Monolayer of confluent PAECs was exposed to laminar shear stress with low pulsation $( \pm 10 \%)$ to mimic normal flow conditions in pulmonary arteries. The flow studies were carried out using a flow chamber connected to a variable speed flow pump (Control Company, Friendswood, Tex., USA). Physiological shear stresses in the arterial vascular network range from 10 to 70 dyn/ $\mathrm{cm}^{2}$. Therefore, we chose 20 and $60 \mathrm{dyn} / \mathrm{cm}^{2}$ as representative mean values for shear stresses in the physiological range, 90 and $120 \mathrm{dyn} / \mathrm{cm}^{2}$ as representative mean values for pathologically high level of shear stresses and $5 \mathrm{dyn} / \mathrm{cm}^{2}$ as representative mean value for pathologically low level of shear stresses. PAEC grown in the absence of flow were also studied. Endothelial cells were exposed to the flow for $20 \mathrm{~h}$, and then collected and analyzed using either Western blot assays or immunofluorescence analyses. A fixed amount of reservoir medium $(15 \mathrm{ml})$ was used for the flow circulation to ensure that the concentrations of released substances were comparable between experiments. The medium was collected and stored at $-80^{\circ} \mathrm{C}$ until use. Two types of flow conditioning were tested: in one case, the flow was increased abruptly to the predefined shear stress level; in the other case, the flow was gradually increased to the predefined shear stress level by preconditioning with $20 \mathrm{dyn} / \mathrm{cm}^{2}$ for $2 \mathrm{~h}$. No significant differences were observed between these 2 conditions. Additionally, as pressure may also modulate pulmonary reactivity [18], the pressure was measured and found to fall in a low pressure range (within the range of normal blood pressure in pulmonary vascular system, $<20 \mathrm{~mm} \mathrm{Hg}$ ). Thus, the pressure influence is not taken into consideration in this study.

To study the signaling pathway that regulates the shear stressinduced endothelial nitric oxide synthase (eNOS) expression and activation, PAECs were incubated with $100 \mathrm{~nm}$ phosphoinositide-3 kinase (PI3K) inhibitor Wortmannin (Sigma, St. Louis, Mo., USA) for $2 \mathrm{~h}$ and then they were exposed to flow shear stress of $20 \mathrm{dyn} / \mathrm{cm}^{2}$ using medium with $100 \mathrm{~nm}$ Wortmannin.

To examine the response of cocultured PAECs and PASMCs to various flow conditions, PASMCs were seeded on microscope slides that were coated with collagen matrix and PAECs were seeded on isopore polycarbonate membranes with $4-\mu \mathrm{m}$ pores (Millipore Corporate, Billerica, Mass., USA) coated with matrigel matrix, a mimetic basement membrane layer (1:10 dilution; BD Biosciences, Franklin Lakes, N.J., USA). During experiments, membranes seeded with PAECs were brought into direct contact with slides seeded with PASMCs before PAECs were subject to flow. 


\section{Immunofluorescence Confocal Imaging}

After cells were exposed to different shear stress for $20 \mathrm{~h}$, slides were fixed with $4 \%$ paraformaldehyde, permeabilized with $0.1 \%$ triton, blocked with FBS, incubated with primary rabbit polyclonal anti-vascular endothelial cadherin (VE-cadherin) antibody (Alexis Biochemicals, San Diego, Calif., USA), followed by incubation with anti-rabbit IgG antibody conjugated with fluorescent dye (Cy3). Subsequently, slides were incubated with phalloidin conjugated with fluorescent dye (Alexa Fluor 488; Invitrogen) to detect F-actin stress fibers, and then mounted with DAPI SlowFade (Invitrogen) to detect cell nuclei. Fluorescently labeled cells were evaluated using an epifluorescence and confocal microscope (Zeiss, Peabody, Mass., USA).

\section{Western Blot Analysis}

Western blot assays were carried out on PAECs after they were exposed to different shear stress conditions. Ten micrograms of each PAEC protein extract were loaded, separated and transferred to a nitrocellulose membrane. The membrane was incubated with polyclonal rabbit eNOS antibody (Santa Cruz Biotechnology Inc., Santa Cruz, Calif., USA; 1:5,000 dilution), polyclonal rabbit phospho-eNOS ${ }^{\text {s1177 }}$ antibody (Cell Signaling, Danvers, Mass., USA; 1:1,000 dilution), polyclonal rabbit Akt antibody (Cell Signaling; 1:500 dilution), polyclonal rabbit phospho-Akt ${ }^{\mathrm{Ser} 473}$ antibody (Cell Signaling; 1:500 dilution), polyclonal rabbit prostaglandin I synthase antibody (Cayman Chemical, Ann Arbor, Mich., USA; 1:5,000 dilution) or polyclonal rabbit anti-vascular endothelial growth factor (VEGF) antibody (Santa Cruz Biotechnology Inc.; 1:100 dilution). The next day, the membrane was incubated with either anti-rabbit or anti-mouse IgG antibody (Amersham Biosciences, Piscataway, N.J., USA), then incubated with electrochemiluminescence reagent (ECL; Amersham Biosciences), and finally exposed to hyperfilm (Amersham Biosciences) for visualization of protein expression.

For PASMCs, blots were incubated with monoclonal mouse proliferating cell nuclear antigen (PCNA) antibody (Sigma; 1:1,000 dilution), monoclonal mouse smooth muscle $\alpha$-actin ( $\alpha$-SM-actin) antibody (Sigma; 1:1,000 dilution), or monoclonal mouse smooth muscle myosin heavy chain (SM-MHC) antibody (Sigma; 1:100 dilution). In the Western blot assays for PAECs and PASMCs, $\beta$-actin or GAPDH was used as reference proteins.

\section{ELISA Assay}

The ET-1 level in the media collected after flow experiments was determined using a commercial endothelin ELISA Kit (American Research Products Inc., Belmont, Mass., USA). In brief, a serial standard was made with standard cell culture medium. A detection antibody was added to all samples and standard wells, and was incubated. The next day, the conjugates and substrate were added to all wells. Absorbance was determined using a plate reader at $450 \mathrm{~nm}$ using $620 \mathrm{~nm}$ as the reference. The results were corrected according to the protein weight and expressed as picomole per microgram of protein.

\section{Enzyme Immunoassay}

Prostacyclin and thromboxane $\mathrm{A}_{2}$ content in the media collected after flow experiment was measured as their stable metabolites, 6-keto-prostaglandin $\mathrm{F}_{1} \alpha\left(\mathrm{PGF}_{1} \alpha\right)$ and thromboxane $\mathrm{B}_{2}$ $\left(\mathrm{TxB}_{2}\right)$, using commercial $\mathrm{PGF}_{1} \alpha$ and $\mathrm{TxB}_{2}$ EIA Kit (Cayman Chemical) following the manufacturer's instruction.

Effects of Pathological Flow on

Pulmonary Endothelial Function
Nitric Oxide Release Assay

Total nitric oxide (NO) content in flow media was determined by measuring total nitrite concentration using Total Nitric Oxide Assay Kit (Thermo Scientific, Rockford, Ill., USA) following the manufacturer's instruction.

\section{Data Analysis}

All data are expressed as means \pm SEM. Comparisons among means of the experimental groups were made using one-way ANOVA and if there was significant difference among groups, Student's t test was used to compare individual groups. $\mathrm{p}<0.05$ was considered significantly different.

\section{Results}

\section{Pathological Shear Stresses Reduce Vasodilator}

Release and Promote Vasoconstrictor Release

Because vasoconstriction is thought to play a major role in the pulmonary hypertension process, we examined the release of both vasodilators (that is, NO and prostacyclin) and vasoconstrictors (that is, endothelin and thromboxane) from the pulmonary arterial endothelium in response to various flow conditions. Figure 1a shows endothelial release of NO. Release was highest at physiological shear stresses, 20 and $60 \mathrm{dyn} / \mathrm{cm}^{2}$. Compared to $20 \mathrm{dyn} / \mathrm{cm}^{2}$, pathologically high shear stress reduced NO release by $14 \%$ at $90 \mathrm{dyn} / \mathrm{cm}^{2}$ and by $15 \%$ at 120 $\mathrm{dyn} / \mathrm{cm}^{2}$ ( $\left.\mathrm{p}<0.05\right)$. Compared to physiological shear stress of $20 \mathrm{dyn} / \mathrm{cm}^{2}$, a low shear stress $\left(5 \mathrm{dyn} / \mathrm{cm}^{2}\right)$ or no shear stress $\left(0 \mathrm{dyn} / \mathrm{cm}^{2}\right)$ also reduced NO release. Figure $1 \mathrm{~b}$ shows release of prostacyclin, an endothelial-derived factor with potent vasodilatory effect. We assessed release of $\mathrm{PGI}_{2}$ in the media by measuring its stable metabolite $\mathrm{PGF}_{1 \alpha}$. $\mathrm{PGF}_{1 \alpha}$ release was optimal at physiological shear stresses (20 and $60 \mathrm{dyn} / \mathrm{cm}^{2}$ ). Compared to physiological shear stress of $20 \mathrm{dyn} / \mathrm{cm}^{2}$, pathologically high shear stress $\left(90\right.$ and $120 \mathrm{dyn} / \mathrm{cm}^{2}$ ) decreased $\mathrm{PGF}_{1 \alpha}$ release by $30-40 \%(\mathrm{p}<0.05)$ and a pathologically low shear stress $\left(5 \mathrm{dyn} / \mathrm{cm}^{2}\right)$ or no shear stress $\left(0 \mathrm{dyn} / \mathrm{cm}^{2}\right)$ also decreased $\mathrm{PGF}_{1 \alpha}$ release by similar percentage $(\mathrm{p}<$ 0.05). Compared to $\mathrm{NO}$ and $\mathrm{PGF}_{1 \alpha}, \mathrm{ET}-1$ release showed a different pattern in relation to flow shear stress. ET-1 production was low at 20,60 and $90 \mathrm{dyn} / \mathrm{cm}^{2}$ (fig. 1c). Pathologically high shear stress increased ET-1 release by $65 \%$ at $120 \mathrm{dyn} / \mathrm{cm}^{2}$, compared to $20 \mathrm{dyn} / \mathrm{cm}^{2}$. ET-1 release at $5 \mathrm{dyn} / \mathrm{cm}^{2}$ was also increased $40 \%$ compared to $20 \mathrm{dyn} / \mathrm{cm}^{2}$. Thromboxane $A_{2}$ is another important vasoconstrictor in pulmonary hypertension. We found that compared to the physiological shear stress $\left(20 \mathrm{dyn} / \mathrm{cm}^{2}\right)$, the content of thromboxane $\mathrm{B}_{2}$, a stable metabolite of $\mathrm{TxA}_{2}$, in the medium was $46 \%$ higher at static condition 


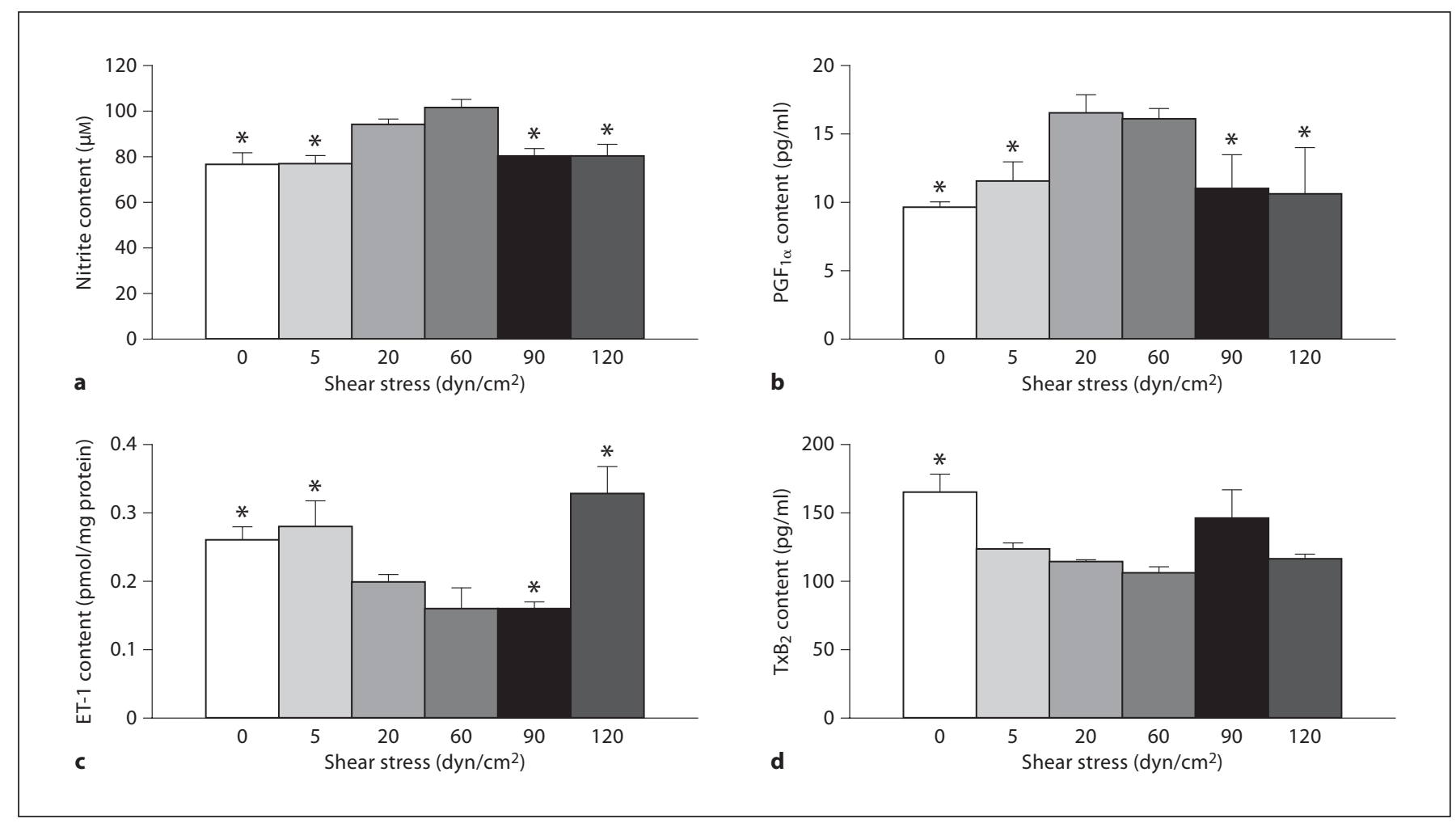

Fig. 1. Pathological shear stresses reduce $\mathrm{NO}$ and $\mathrm{PGF}_{1 \alpha}$ release and promote ET-1 release. a Nitric oxide release as measured by the total content of nitrite in flow media. b EIA measurement results of the $\mathrm{PGF}_{1} \alpha$ content in the flow media. c ELISA measurement results of the ET-1 content in the flow media. d EIA mea-

surement results of the $\mathrm{TxB}_{2}$ content in the flow media. The medium was collected from the flow circulation after cells were exposed to different shear stresses. Data represent means \pm SEM $(\mathrm{n}=3-6) .{ }^{*} \mathrm{p} \leq 0.05$ compared to $20 \mathrm{dyn} / \mathrm{cm}^{2}$ shear stress.

$(\mathrm{p}<0.05)$. Neither low $\left(5 \mathrm{dyn} / \mathrm{cm}^{2}\right)$ nor high pathological flow shear stresses $\left(90\right.$ and $120 \mathrm{dyn} / \mathrm{cm}^{2}$ ) affected $\mathrm{TxB}_{2}$ concentrations compared to physiological shear (20 dyn/ $\mathrm{cm}^{2} ; 0.17<\mathrm{p}<0.5$; fig. 1d).

\section{Shear Stresses Affect NO Release by Altering \\ Expression and Activation of eNOS through the \\ PI3K/Akt Signaling Pathway}

Because NO release was found to be significantly regulated by flow, we sought to determine mechanisms involved in this process. We examined changes in eNOS expression and activation in PAECs in response to shear stress and then studied the PI3K/Akt pathway in relation to changes in eNOS. Compared to physiological shear stress conditions, total eNOS (T-eNOS) expression at shear stress above or below the physiological range was decreased (fig. 2a). It was decreased by $24 \%$ at $90 \mathrm{dyn} / \mathrm{cm}^{2}$ and by $31 \%$ at $120 \mathrm{dyn} / \mathrm{cm}^{2}(\mathrm{p}<0.05)$. Pathologically low shear stress $\left(5 \mathrm{dyn} / \mathrm{cm}^{2}\right)$ also decreased T-eNOS protein

expression by $65 \%$, compared to physiological shear stress of $20 \mathrm{dyn} / \mathrm{cm}^{2}$ (fig. 2a). We found that expression of TeNOS at all flow conditions were higher than those under static conditions $(0.03 \pm 0.02)$, which is in good agreement with previous studies [19].

We also investigated shear stress-mediated activation of eNOS by examining phosphorylation of eNOS. The expression of phosphorylated-eNOS (P-eNOS) at S1179, expressed as the ratio of P-eNOS to T-eNOS, was highest at $20 \mathrm{dyn} / \mathrm{cm}^{2}(0.62 \pm 0.09)$. Phosphorylation of eNOS was dramatically decreased by 87 and $82 \%$ at 90 and $120 \mathrm{dyn} / \mathrm{cm}^{2}$, respectively $(0.08 \pm 0.01$ and $0.11 \pm$ 0.01 , respectively; $\mathrm{p}<0.05$ compared to $\left.20 \mathrm{dyn} / \mathrm{cm}^{2}\right)$. Phosphorylation of eNOS at $5 \mathrm{dyn} / \mathrm{cm}^{2}(0.51 \pm 0.03 ; \mathrm{p}=$ 0.3608 ) was not statistically different from $20 \mathrm{dyn} / \mathrm{cm}^{2}$ (fig. 2b). However, because T-eNOS expression was far lower at $5 \mathrm{dyn} / \mathrm{cm}^{2}$ than that at $20 \mathrm{dyn} / \mathrm{cm}^{2}$, the amount of P-eNOS was actually lower at $5 \mathrm{dyn} / \mathrm{cm}^{2}$ as well. Phosphorylation of eNOS was dramatically reduced under 


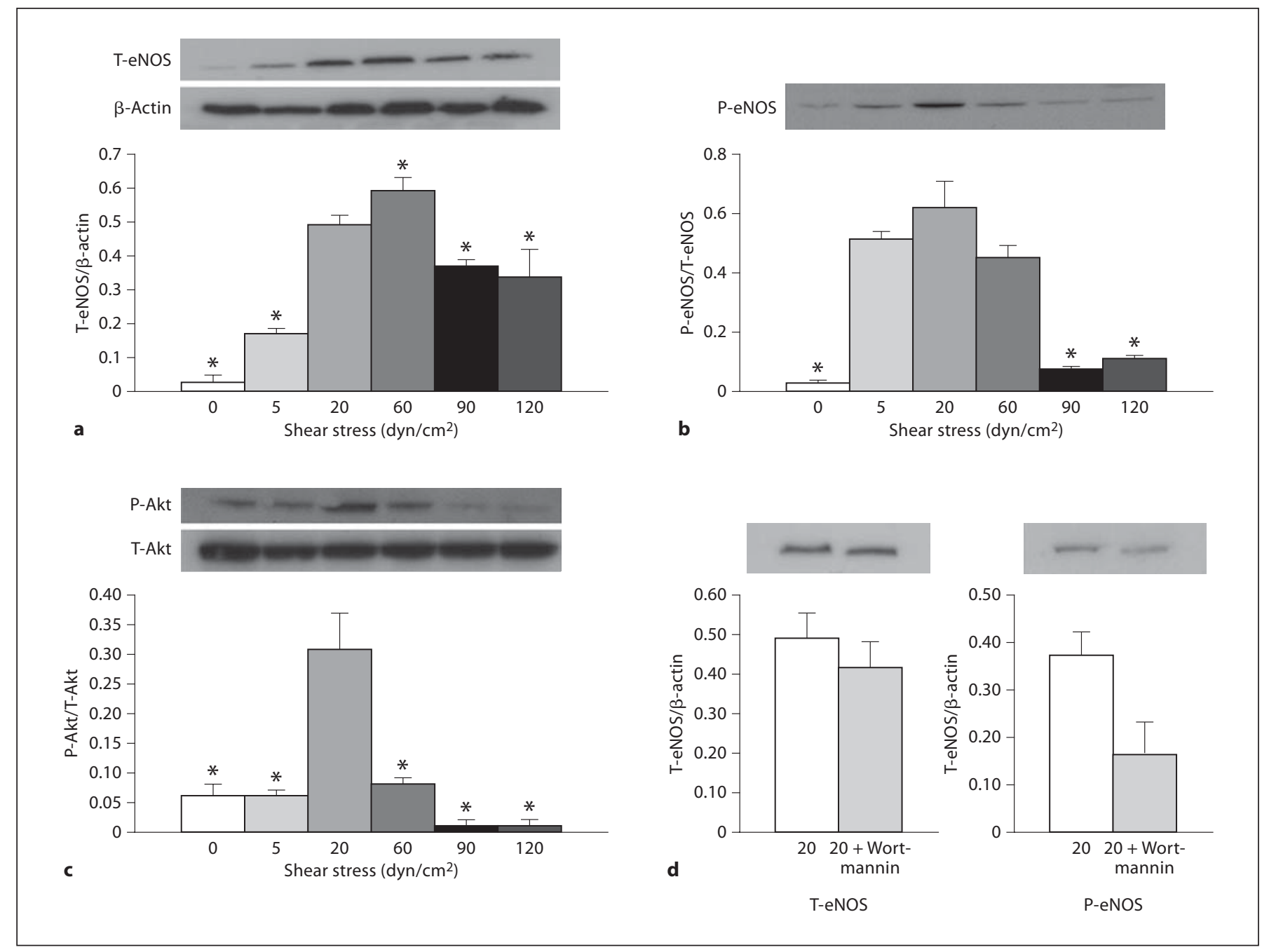

Fig. 2. Shear stresses affect expression and activation of eNOS through PI3K/Akt signaling pathway: Western blot images and quantitative measurements of proteins expressed by PAECs. a TeNOS expressed as the ratio of T-eNOS to $\beta$-actin. $\mathbf{b}$ P-eNOS expressed as the ratio of P-eNOS to T-eNOS. c Phosphorylated $\mathrm{Akt}^{\mathrm{Ser} 473}$ (P-Akt) expressed as the ratio of P-Akt to total Akt
(T-Akt). Protein expressions were measured with PAECs exposed to shear stresses of $0,5,20,60,90$ and $120 \mathrm{dyn} / \mathrm{cm}^{2}$. d T-eNOS and P-eNOS expression under flow of $20 \mathrm{dyn} / \mathrm{cm}^{2}$ with or without Wortmannin $(100 \mathrm{nM})$. Data represent means \pm SEM $(\mathrm{n}=$ 4-6 for each group). ${ }^{*} \mathrm{p} \leq 0.05$ compared to shear stress at $20 \mathrm{dyn} / \mathrm{cm}^{2}$. static conditions $(0.03 \pm 0.01)$ compared to both 5 and $20 \mathrm{dyn} / \mathrm{cm}^{2}$.

Akt has been demonstrated as a critical signaling intermediate in the shear-dependent regulation of eNOS. Akt is a serine/threonine kinase activated by several phosphatidylinositol-dependent protein kinases. Phosphorylation of S473 on Akt is coincident with Akt activation in vivo and in vitro and has been used as a marker for Akt activity. Therefore, we examined phosphorylation of S473 on Akt in PAECs exposed to various flow conditions. As demonstrated in figure $2 c$, phosphoryla- tion of Akt, expressed as the ratio of phosphorylated Akt to total Akt, was highest at physiological shear stress of $20 \mathrm{dyn} / \mathrm{cm}^{2}$ (0.31 \pm 0.06$)$. Phosphorylation of Akt was decreased by $74 \%$ at $60 \mathrm{dyn} / \mathrm{cm}^{2}$, and dramatically decreased by $97 \%$ at 90 and $120 \mathrm{dyn} / \mathrm{cm}^{2}$. Phosphorylation of Akt was also decreased by $81 \%$ at $5 \mathrm{dyn} / \mathrm{cm}^{2}(0.06 \pm$ $0.01 ; \mathrm{p}<0.05)$ and static conditions $(0.06 \pm 0.02 ; \mathrm{p}<$ $0.05)$. Our results showed that shear stress-mediated phosphorylation of Akt was generally consistent with changes of eNOS phosphorylation in response to flow shear stresses. 


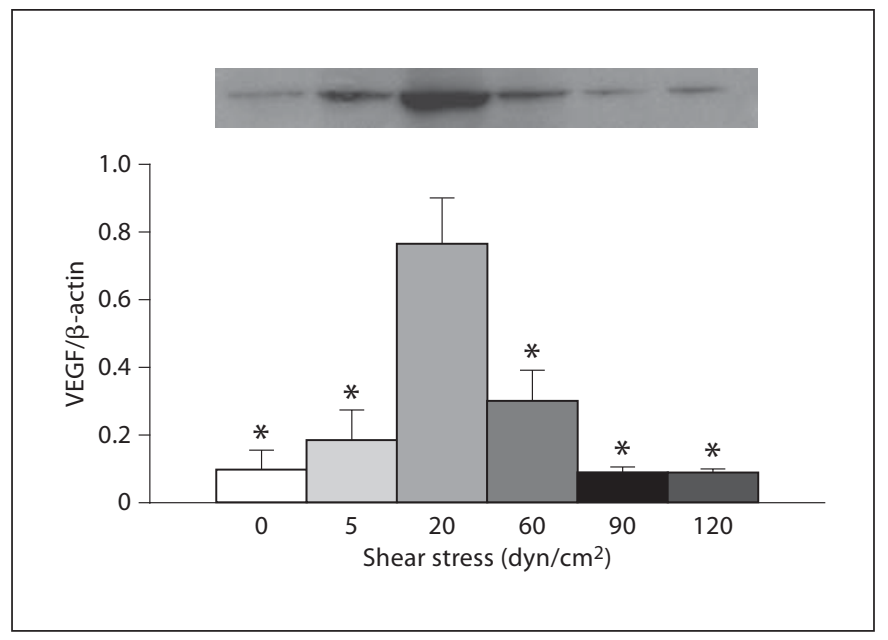

Fig. 3. Pathological shear stresses reduce VEGF expression: Western blot images and quantitative measurements of VEGF expression. Results expressed as ratio of VEGF to $\beta$-actin. Data represent means $\pm \mathrm{SE}(\mathrm{n}=3-4) .{ }^{*} \mathrm{p} \leq 0.05$ compared to $20 \mathrm{dyn} /$ $\mathrm{cm}^{2}$.

To confirm the signaling pathway that regulates eNOS expression under flow stimulation we incubated PAECs with $100 \mathrm{~nm}$ Wortmannin, a PI3K inhibitor, and then exposed cells to flow shear stress of $20 \mathrm{dyn} / \mathrm{cm}^{2}$. We found that without flow shear stress, Wortmannin had no effect on either T-eNOS expression or eNOS phosphorylation (not shown). After exposure to shear stress, though expression of T-eNOS was not changed, phosphorylation of eNOS was significantly decreased by $54 \%(\mathrm{p}<0.05)$ in the presence of Wortmannin (fig. 2d).

\section{Effects of Flow Shear Stresses on VEGF Expression}

VEGF is an important growth factor and signaling protein that is associated with endothelial growth, vasculogenesis and angiogenesis, and it is essential in maintaining normal endothelial function. Thus, we explored changes in VEGF expression by PAECs in response to different flow conditions. As shown in figure 3, VEGF protein expression, expressed as ratio of VEGF to $\beta$-actin, was highest at physiological shear stress of $20 \mathrm{dyn} /$ $\mathrm{cm}^{2}(0.77 \pm 0.13)$. Its expression was decreased by more than $50 \%$ at $60 \mathrm{dyn} / \mathrm{cm}^{2}$. At shear stresses of 90 and 120 dyn $/ \mathrm{cm}^{2}$, VEGF expression was further decreased. Pathologically low shear stress at $5 \mathrm{dyn} / \mathrm{cm}^{2}$ also dramatically reduced VEGF protein expression. VEGF expression was extremely low under static conditions $(0.09 \pm 0.06)$.

\section{Effects of Flow Shear Stresses on the Endothelial}

\section{Structure}

Endothelial cells sense flow conditions through mechanosensory complexes. Cytoskeletal proteins and VEcadherin have been described as structural constituents of large mechanosensory complexes in endothelial cells $[12,20]$. A relationship between these structural molecules and Akt/eNOS module has been suggested in previous studies. Thus, we examined effects of flow on the distribution of F-actin and VE-cadherin (fig. 4). Staining with F-actin antibody showed that F-actin aligned in the direction of the flow. The brightness of staining and the pattern of F-actin stress fibers in endothelial cells were not significantly affected by the level of the shear stress, though they were consistently different than those in endothelial cells cultured under static conditions. In contrast, the magnitude of shear stress regulated the brightness of staining and distribution of VE-cadherin. Under static or low flow conditions $\left(5 \mathrm{dyn} / \mathrm{cm}^{2}\right)$, endothelial cells displayed continuous VE-cadherin staining along the peripheral cell membrane. After exposure to physiological shear stress conditions with 20 and $60 \mathrm{dyn} / \mathrm{cm}^{2}$, endothelial cells still displayed continuous VE-cadherin staining along the peripheral cell membrane, while the staining in the center of basal and apical membranes was stronger and displayed punctuate accumulations. Discontinuous VE-cadherin was found on the cells exposed to shear stresses of 90 and $120 \mathrm{dyn} / \mathrm{cm}^{2}$. VE-cadherin staining along the peripheral cell membrane became intermittent and predominantly exhibited small, distinct 'dashes' rather than continuous linear staining. This suggested disruption of cell-cell junctions by pathologically high flow.

\section{Effects of Shear Stresses on Cocultured PASMC}

Proliferation and Contractile Protein Expression

Endothelial cells serve as mechanical sensors that transduce mechanical signals to biochemical signals which can affect other cells or the blood vessel wall. Because pulmonary vascular diseases are often characterized by changes in SMC contractile activity and proliferation, we studied endothelial-mediated shear stress effects on PASMC contractile protein expression and proliferation in coculture experiments. We examined 2 contractile proteins of SMCs, $\alpha$-SM-actin and SM-MHC, and the proliferation marker PCNA. As shown in figure 5, expression of $\alpha$-SM-actin and SM-MHC was significantly increased at high shear stress level $\left(90\right.$ and $\left.120 \mathrm{dyn} / \mathrm{cm}^{2}\right)$ compared to that at physiological shear stress $\left(20 \mathrm{dyn} / \mathrm{cm}^{2}\right)$. Shear stress did not have significant effects on smooth muscle proliferation as assessed by PCNA (fig. 5c). 
0
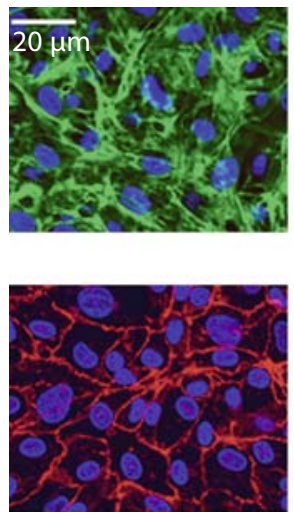

5
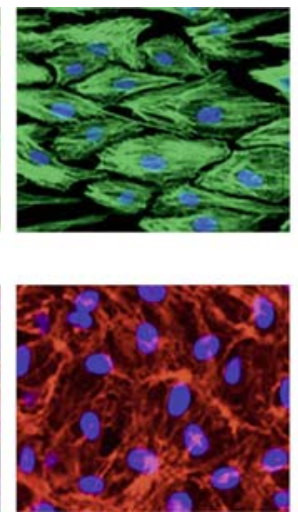

20
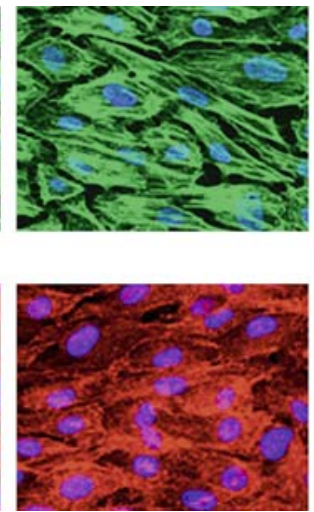

60
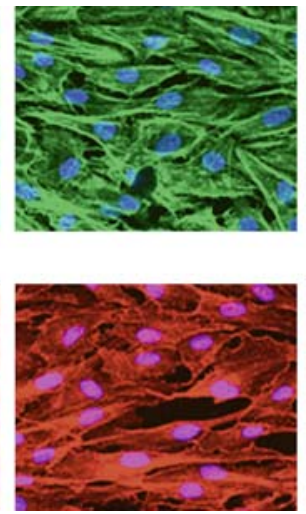

90
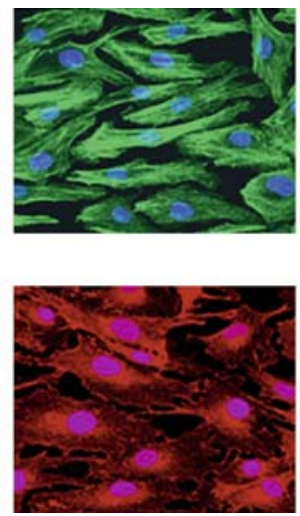

120
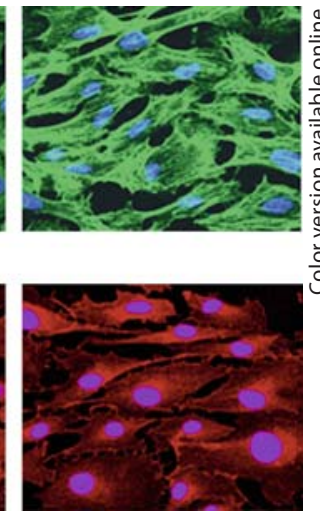

Flow direction

Fig. 4. Shear stresses affect endothelial structure. Subcellular structures of PAECs were stained with different colors: F-actin (green), VE-cadherin (red) and nuclei (blue). The numbers indicate the level of flow shear stresses $\left(\mathrm{dyn} / \mathrm{cm}^{2}\right)$ that cells were exposed to.

Fig. 5. High shear stresses increase $\alpha$-SMactin and SM-MHC expression, but not DNA synthesis in cocultured PASMC: quantitative measurements of $\alpha$-SM-actin (a), and SM-MHC (b) in PASMCs cocultured with PAECs. c Western blot images of $\alpha$-SM-actin, SM-MHC and PCNA expressions in PASMCs cocultured with PAECs. Data represent means $\pm \mathrm{SE}$ ( $\mathrm{n}=3-4) .{ }^{*} \mathrm{p} \leq 0.05$ compared to $20 \mathrm{dyn} /$ $\mathrm{cm}^{2}$.
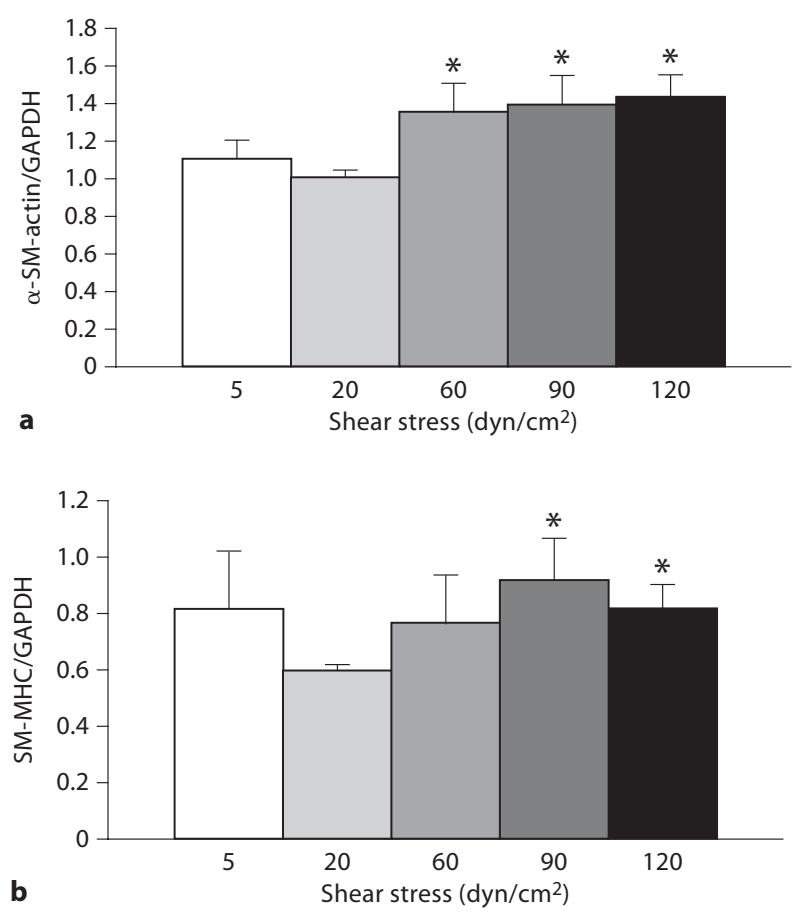

Shear stress $\left(\mathrm{dyn} / \mathrm{cm}^{2}\right)$

5

20

60

90

120

$\alpha$-SM-actin

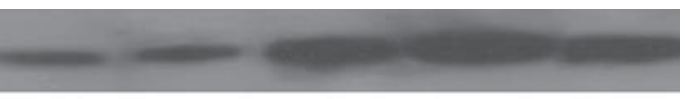

SM-MHC

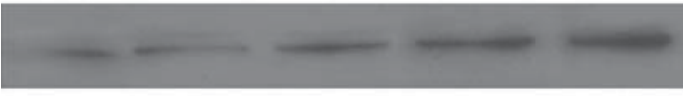

PCNA

c 


\section{Discussion}

Many forms of pulmonary hypertension are associated with significant increases and/or decreases in blood flow and shear stress in the pulmonary circulation. To better understand the effects of shear on endothelial production of vasoactive mediators and growth factors in the pulmonary circulation, in the context of their potential contribution to the hypertensive process, we devised an artificial flow system in which the effects of a wide range of shear stresses on endothelium and endothelium-SMC interactions could be assessed. Our results reveal that: (1) compared to physiological flow shear stresses (20 and $60 \mathrm{dyn} / \mathrm{cm}^{2}$ ), pathologically high (90 and $120 \mathrm{dyn} / \mathrm{cm}^{2}$ ) and pathologically low $\left(5 \mathrm{dyn} / \mathrm{cm}^{2}\right)$ flow shear stresses attenuated PAEC production of vasodilation- and angiogenesis-related molecules such as $\mathrm{NO}, \mathrm{PGI}_{2}$ and VEGF, and enhanced release of vasoconstrictor ET-1, (2) physiological flow activated Akt/eNOS pathway while pathological flow depressed this signaling pathway, (3) pathologically high flow redistributed and weakened the staining of peripheral VE-cadherin junctions, and (4) pathologically high and low flow conditions imposed on endothelial cells upregulated $\alpha$-SM-actin and SM-MHC expression in the underlying SMCs, but did not change smooth muscle proliferation. Collectively, these findings are consistent with the high vascular resistance observed in patients and animals with high flow.

While the effects of hemodynamic shear stresses on the vascular endothelium have been extensively studied and the resulting findings associated with pathogenesis of atherosclerosis and coronary heart diseases in the systemic circulation, few studies have examined whether and how shear stresses play a role in the endothelial dysfunction which contributes to pulmonary vascular diseases [2]. Results from atherosclerosis-related flow studies in the systemic circulation might not be directly related to hemodynamic effects in the pulmonary circulation, since it is increasingly appreciated that endothelial phenotypes and functional responses are organ specific [21]. The flow conditions and primary cells used in the present study are specific to the pulmonary circulation, and the results are consistent with a potentially dual contribution of changes in flow magnitude to the pathogenesis of pulmonary vascular diseases [10]. Pathologically high flow conditions were studied because acute increase in pulmonary blood flow is an integral part of many congenital heart defects which often lead to endothelial dysfunction and pulmonary vascular diseases [22]. Pathologically low flow conditions were studied be- cause chronic reduction of blood flow and flow shear stress have also been observed in certain conditions and might have long-term effects on pulmonary vascular disease [23]. Assays on the molecules related to key vascular tone mediators such as $\mathrm{NO}, \mathrm{PGI}_{2}, \mathrm{ET}-1$ and $\mathrm{TxA}_{2}$ were studied because they are associated with regulation of vascular tone in the disease conditions. Coculture studies were performed to better understand endothelial-SMC interactions under varying flow conditions.

With regard to existing knowledge about flow-mediated effects on the endothelium, several findings in the present study merit discussion. First, prior work has established that flow-induced relaxation is an endothelialmediated response. We also found that physiologically normal shear maximized vasodilator release and that pathologically low flow reduced endothelial release of vasodilators. The unique finding of the present study was that the production of $\mathrm{NO}$ and $\mathrm{PGI}_{2}$ did not increase monotonically and that the ET- 1 and $\mathrm{Tx}_{2}$ release did not decrease monotonically with the increase of flow. Our results suggested that both pathologically high and low shear stresses lead to an increased vasoconstrictor (that is, ET- 1 and $\mathrm{TxA}_{2}$ ) to vasodilator (that is, $\mathrm{NO}, \mathrm{PGI}_{2}$ ) ratio. NO, $\mathrm{PGI}_{2}, \mathrm{ET}-1$ and $\mathrm{TxA}_{2}$ are important vasoactive substances and the balance among them largely determines vascular tone and platelet aggregation. The flow-mediated effects demonstrated here under pathological shear stress conditions may partly contribute to prolonged vasoconstriction and enhanced vascular resistance observed in flow-induced pulmonary hypertension. This result is consistent with a previous study which shows that eNOS expression is decreased in PAECs isolated from animals with high-flow pulmonary shunts [6].

Though it has been established that flow modulates eNOS expression, the underlying signaling pathways involved remain unclear and controversial. Various signaling pathways can promote NO release. There have been conflicting results as to which protein kinases - protein kinase A, protein kinase B (Akt), other Ser/Thr protein kinases or tyrosine kinases - are responsible for sheardependent eNOS regulation [24]. Several in vitro studies showed that positive-mean pulsatile flow shear stress induced eNOS and Akt activity in several endothelial cell lines $[25,26]$. In contrast, Boo et al. [27] showed that laminar shear stress stimulates phosphorylation of eNOS by protein kinase A but not Akt. Our results in bovine PAECs demonstrate that the Akt/eNOS pathway is involved in flow-mediated eNOS expression and that its activation is dependent on the flow shear stress magnitude. 
However, the fold changes of reduction at 5 and 60 dyn/ $\mathrm{cm}^{2}$ compared to $20 \mathrm{dyn} / \mathrm{cm}^{2}$ were much higher for phosphorylated Akt than for P-eNOS. This suggests that though the Akt/eNOS module is a major signaling pathway that contributes to flow-activated eNOS expression, other pathways are likely involved in eNOS activation, particularly in the range of low and physiological flow shear stresses. ERK-1 and MAPK, for example, have been demonstrated to be involved in the flow-mediated eNOS activation. The role of signaling pathways in regulating eNOS needs further evaluation.

The demonstration of structural changes in PAEC might partly explain the functional changes observed. It shows that different sensing mechanisms might be involved in pulmonary vascular endothelial responses to the 2 types of pathological flow conditions (low and high flow shear stresses) studied. The important questions raised by these observations are interesting and deserve in-depth exploration in the future. The vascular endothelial cells serve as an integrated barrier between blood and underlying SMCs. They also act as important mechanosensory complexes to sense hemodynamic forces and translate mechanical to molecular signals. As demonstrated in previous studies [20, 28, 29], both Factin and VE-cadherin can act as structural constituents of large mechanosensory complexes, and involve the PI3K/Akt pathway in endothelial cells. VE-cadherin is part of multicomponent mechanosensory complexes which transmit forces and activate signaling pathways $[20,28]$. The redistribution of VE-cadherin under flow conditions was previously reported in cell culture and in vivo. Additionally, VE-cadherin was shown to be involved in the activation of Akt by flow [28]. In agreement with these studies, we showed that flow induced redistribution of VE-caderin. Our data showed an interesting and unique finding that, compared to physiological flow conditions, no flow or pathologically low flow exerts effects on the cell cytoskeleton (F-actin), while pathologically high flow conditions exert more influence on the cell-cell junctions. Discontinuous VE-cadherin expression was found on the cells exposed to shear stresses of 90 and $120 \mathrm{dyn} / \mathrm{cm}^{2}$. This suggests that VEcadherin expression was deleteriously affected by these high-flow conditions and might contribute to the altered protein expression observed. Our data also support the involvement of VE-cadherin in the activation of Akt by showing phosphorylation of Akt decreased along with disruption of VE-cadherin junction under high-flow conditions.

Effects of Pathological Flow on

Pulmonary Endothelial Function
Previous studies have suggested that the phenotypic changes in PASMCs observed in high-flow pulmonary hypertension models were induced by factors released by the endothelium. Decrease in vasodilator release and increase in vasoconstrictor release by PAECs under pathological flow conditions will likely increase vascular tone. Our results, showing that high shear stress leads to changes in the production of these endothelial factors that increase PASMC expression of the contractile proteins $\alpha$ SM-actin and SM-MHC, would provide an explanation of increased tone and contractile responses in vessels exposed to prolonged pathophysiological flow conditions. In contrast to other observations [30], we found that the flow magnitude did not influence PASMC proliferation when cultured with PAECs exposed to pathological flow conditions. This is somewhat surprising, since the NO and $\mathrm{PGI}_{2}$ release decreased and the ET-1 release increased at high shear stress. It is known that prostacyclin and $\mathrm{NO}$ suppresses smooth muscle proliferation and endothelin can act as a mitogen under certain conditions. But as shown in a recent paper by Bakker et al. [31], altered blood flow may trigger an inflammatory response that facilitates vessel remodeling, and vascular tone is a crucial determinant of the direction of the remodeling response. In the model system used, pathological flow on endothelial cells did not result in the production of a factor whose net effect was to increase SMC proliferation, but rather in the production of a factor(s) that increased contractile protein expression in SMC. The identity of this factor(s) remains to be determined.

Several limitations of the study are acknowledged. First, the particular values of flow shear stress chosen in this study $\left(5,20,60,90\right.$ and $\left.120 \mathrm{dyn} / \mathrm{cm}^{2}\right)$ are not absolutely representative of low-pathological, normal and high-pathological shear values seen in the pulmonary vasculature. These values, especially the larger ones, were chosen based on patient-specific computational modeling studies of the pulmonary vasculature in children with pulmonary arterial hypertension, but certainly may not represent the entire range of normal or high shear values that may be seen in this highly heterogeneous population. However, the particular patterns of cell response between lower and higher values of shear suggest strong correlations between pathologically high shear and abnormal cell response; these will undoubtedly be clarified by additional studies linking patientspecific measurements of pulmonary vascular hemodynamics and in vitro shear studies. Also, in our studies, endothelial cells were cultured on a fibronectin-coated stiff substrate. The rigidity of the system is different than

J Vasc Res 2009;46:561-571 569 
the actual interactions between vascular endothelial cells and the basement membrane in vivo. Further, the effect of vascular SMCs on vascular endothelial cell responses was not considered in this study because in our coculture studies they were separated by a polycarbonate membrane and endothelial-SMC contact did not reflect that in vivo.

In conclusion, present studies show that physiological flow shear stress activates the Akt/eNOS pathway, favors the release of vasodilators and endothelial growth factors, and inhibits the release of vasoconstrictors from PAECs. In contrast, either high or low pathological flow shear stress suppress the Akt/eNOS pathway, decrease the ratio of vasodilators to vasoconstrictors produced from endothelial cells, and enhance expression of contractile proteins in underlying SMCs. These findings suggest flow is a critical factor in the regulation of pulmonary vascular cell physiology and pathology.

\section{Acknowledgements}

The authors would like to acknowledge Sandra Walchak from the CVP group for providing bovine PAECs and PASMCs in this study. Also, we would like to thank Lila Sisbarro and Aaron Richman for their help in the study, and Craig Lanning and Dr. Kendall Hunter for their insightful input into the study.

This study was funded in part by a grant from the Children's Hospital at Denver (Research Scholar Development Award to W.T.) and the NIH (HL 072738 to R.S., HL 084923 to K.R.S. and HL $14985-35$ to K.R.S.).

\section{References}

1 Dakshinamurti S: Pathophysiologic mechanisms of persistent pulmonary hypertension of the newborn. Pediatr Pulmonol 2005;39: 492-503.

-2 Cool CD, Groshong SD, Oakey J, Voelkel NF: Pulmonary hypertension: cellular and molecular mechanisms. Chest 2005;128:565S$571 \mathrm{~S}$.

-3 Botney MD: Role of hemodynamics in pulmonary vascular remodeling: implications for primary pulmonary hypertension. Am J Respir Crit Care Med 1999;159:361-364.

$\checkmark 4$ Ben Driss A, Devaux C, Henrion D, Duriez M, Thuillez C, Levy BI, Michel JB: Hemodynamic stresses induce endothelial dysfunction and remodeling of pulmonary artery in experimental compensated heart failure. Circulation 2000;101:2764-2770.

5 Storme L, Parker TA, Kinsella JP, Rairigh RL, Abman SH: Chronic hypertension impairs flow-induced vasodilation and augments the myogenic response in fetal lung. Am J Physiol Lung Cell Mol Physiol 2002; 282:L56-L66

6 Ross GA, Oishi P, Azakie A, Fratz S, Fitzgerald RK, Johengen MJ, Harmon C, HendricksMunoz K, Xu J, Black SM, Fineman JR: Endothelial alterations during inhaled NO in lambs with pulmonary hypertension: implications for rebound hypertension. Am J Physiol Lung Cell Mol Physiol 2005;288: L27-L35.

$\checkmark 7$ Rondelet B, Kerbaul F, Van Beneden R, Hubloue I, Huez S, Fesler P, Remmelink M, Brimioulle S, Salmon I, Naeije R: Prevention of pulmonary vascular remodeling and of decreased BMPR-2 expression by losartan therapy in shunt-induced pulmonary hypertension. Am J Physiol Heart Circ Physiol 2005;289:H2319-H2324.
8 Steinhorn R, Russell J, Lakshminrusimha S, Gugino S, Black S, Fineman J: Altered endothelium-dependent relaxations in lambs with high pulmonary blood flow and pulmonary hypertension. Am J Physiol Heart Circ Physiol 2001;280:H311-H317.

-9 Hunter K, Lanning C, Zhang Y, Garg R, Ivy D, Shandas R: Simulations of congenital defect closure and drug reactivity testing in patient-specific models of the pediatric pulmonary vasculature: a 3D numerical study with fluid-structure interaction. J Biomech Eng 2006;128:564-572.

10 Rabinovitch M, Konstam MA, Gamble WJ, Papanicolaou N, Aronovitz MJ, Treves S, Reid L: Changes in pulmonary blood flow affect vascular response to chronic hypoxia in rats. Circ Res 1983;52:432-441.

$\checkmark 11$ Malek AM, Alper SL, Izumo S: Hemodynamic shear stress and its role in atherosclerosis. JAMA 1999;282:2035-2042.

12 Davies PF, Spaan JA, Krams R: Shear stress biology of the endothelium. Ann Biomed Eng 2005;33:1714-1718.

13 Helmlinger G, Geiger RV, Schreck S, Nerem RM: Effects of Pulsatile Flow on Cultured Vascular Endothelial Cell Morphology. J Biomech. Eng 1991;113:123-131.

14 Garcia-Cardena G, Comander J, Anderson KR, Blackman BR, Gimbrone MA, Jr: Biomechanical activation of vascular endothelium as a determinant of its functional phenotype. Proc Natl Acad Sci USA 2001;98: 4478-4485.

15 White C, Frangos J: The shear stress of it all: the cell membrane and mechanochemical transduction. Philos Trans R Soc Lond B Biol Sci 2007;362:1459-1467.
16 Stiebellehner L, Frid M, Reeves J, Low R, Gnanasekharan M, Stenmark K: Bovine distal pulmonary arterial media is composed of a uniform population of well-differentiated smooth muscle cells with low proliferative capabilities. Am J Physiol Lung Cell Mol Physiol 2003;285:819-828.

17 Frid M, Kale V, Stenmark K: Endothelialmesenchymal transdifferentiation: In vitro analysis mature vascular endothelium can give rise to smooth muscle cells. Circ Res 2002;90:1189-1196.

18 Tourneux P, Chester M, Grover T, Abman SH: Fasudil inhibits the myogenic response in the fetal pulmonary circulation. Am J Physiol Heart Circ Physiol 2008;295:H1505H1513.

19 Wedgwood S, Mitchell CJ, Fineman JR, Black SM: Developmental differences in the shear stress-induced expression of endothelial NO synthase: changing role of AP-1. Am J Physiol Lung Cell Mol Physiol 2003;284: L650-L662.

20 Tzima E, Irani-Tehrani M, Kiosses WB, Dejana E, Schultz DA, Engelhardt B, Cao G, DeLisser H, Schwartz MA: A mechanosensory complex that mediates the endothelial cell response to fluid shear stress. Nature 2005; 437:426-431.

21 Aird W: Phenotypic heterogeneity of the endothelium: I. Structure, function, and mechanisms. Circ Res 2007;100:158-173.

22 Steinhorn RH, Russell JA, Lakshminrusimha S, Gugino SF, Black SM, Fineman JR: Altered endothelium-dependent relaxations in lambs with high pulmonary blood flow and pulmonary hypertension. Am J Physiol Heart Circ Physiol 2001;280:H311H317. 
23 Rosenberg AA, Kinsella JP, Abman SH: Cerebral hemodynamics and distribution of left ventricular output during inhalation of nitric oxide. Crit Care Med 1995;23:13911397.

24 Boo YC, Jo H: Flow-dependent regulation of endothelial nitric oxide synthase: role of protein kinases. Am J Physiol Cell Physiol 2003; 285:C499-C508.

25 Kadohama T, Nishimura K, Hoshino Y, Sasajima T, Sumpio BE: Effects of different types of fluid shear stress on endothelial cell proliferation and survival. J Cell Physiol 2007;212:244-251.
26 Ziegler T, Bouzourene K, Harrison VJ, Brunner HR, Hayoz D: Influence of oscillatory and unidirectional flow environments on the expression of endothelin and nitric oxide synthase in cultured endothelial cells. Arterioscler Thromb Vasc Biol 1998;18:686692.

27 Boo YC: Shear stress stimulates phosphorylation of protein kinase A substrate proteins including endothelial nitric oxide synthase in endothelial cells. Exp Mol Med 2006;38: 63-71.

28 Shay-Salit A, Shushy M, Wolfovitz E, Yahav $\mathrm{H}$, Breviario F, Dejana E, Resnick N: VEGF receptor 2 and the adherens junction as a mechanical transducer in vascular endothelia cells. Proc Natl Acad Sci USA 2002;99:94629467.
29 Zhao M, Bai H, Wang E, Forrester J, McCaig C: Electrical stimulation directly induces pre-angiogenic responses in vascular endothelial cells by signaling through VEGF receptors. J Cell Sci 2004; 117:397-405.

30 Buus CL, Pourageaud F, Fazzi GE, Janssen G, Mulvany MJ, De Mey JG: Smooth muscle cell changes during flow-related remodeling of rat mesenteric resistance arteries. Circ Res 2001;89:180-186.

31 Bakker EN, Matlung HL, Bonta P, de Vries CJ, van Rooijen N, Vanbavel E: Blood flowdependent arterial remodelling is facilitated by inflammation but directed by vascular tone. Cardiovasc Res 2008;78:341-348. 